\title{
Multi-frequency study of the B3 VLA sample
}

\section{74-MHz flux densities from VLA A-array data ${ }^{\star}$}

\author{
K.-H. Mack ${ }^{1,2}$, M. Vigotti ${ }^{1}$, L. Gregorini ${ }^{1,3}$, U. Klein ${ }^{2}$, W. Tschager ${ }^{4}$, R. T. Schilizzi ${ }^{5,4}$, and I. A. G. Snellen ${ }^{4}$ \\ 1 INAF - Istituto di Radioastronomia, via Gobetti 101, 40129 Bologna, Italy \\ e-mail: mack@ira.cnr.it \\ 2 Radioastronomisches Institut der Universität Bonn, Auf dem Hügel 71, 53121 Bonn, Germany \\ 3 Dipartimento di Fisica, Università di Bologna, via Irnerio 46, 40126 Bologna, Italy \\ ${ }^{4}$ Sterrewacht Leiden, Postbus 9513, 2300 RA Leiden, The Netherlands \\ 5 International SKA Project Office, Postbus 2, 7990 AA Dwingeloo, The Netherlands
}

Received 28 December 2004 / Accepted 15 February 2005

\begin{abstract}
We present 74-MHz data of 365 B3 VLA sources, which were obtained as a by-product of the observations of Tschager et al. (2003, A\&A, 402, 171) who observed a large area of sky with the VLA in A-array. Apart from the lowestfrequency observations of B3 VLA sources performed so far contributing to our on-going multi-frequency study of this survey, these A-array data also provide the first morphological information at this low frequency. The most intriguing result is the discovery of two new Giant Radio Galaxy candidates, B3 1232+397B at a redshift of 3.22 and B3 1419+419 at a redshift of 0.367. This means that B3 1232+397B would be the most distant GRG known to date. At the same time, these two objects are two new examples of sources with recurrent activity. Our results give a foretaste of the impact which extremely low-frequency radio continuum observations will have for the study of source evolution.
\end{abstract}

Key words. galaxies: active - radio continuum: galaxies - surveys - galaxies: evolution

\section{Introduction}

The B3 VLA survey (Vigotti et al. 1989) consists of 1049 sources selected from the B3 survey (Ficarra et al. 1985) in five flux density bins between declination $37^{\circ}$ and $47^{\circ}$ avoiding the galactic plane. It has been designed to be complete down to flux densities of $100 \mathrm{mJy}$ at $408 \mathrm{MHz}$. In the last years a lot of effort has been spent to enlarge the data base of multi-frequency measurements of this sample. In addition to the original flux densities at $408 \mathrm{MHz}$ the entire classical radio frequency range has been covered for most of the sources in the sample, both from already existent surveys $(6 \mathrm{C}$ at $151 \mathrm{MHz}$, WENSS at $325 \mathrm{MHz}, \mathrm{NVSS}$ at $1400 \mathrm{MHz}, \mathrm{GB} 6$ at $4850 \mathrm{MHz}$ ) and from dedicated observations, mainly at higher frequencies (2.7 GHz, Klein et al. 2003; 4.85 and $10.5 \mathrm{GHz}$, Vigotti et al. 1999, Gregorini et al. 1998).

For specific applications further measurements have been obtained at higher radio frequencies $(32 \mathrm{GHz}$, Mack et al., in prep.; $230 \mathrm{GHz}$, Klein et al. 1995). With the flux density measurements over this large frequency range the majority of

* Tables 2 and 3 are only available in electronic form at the CDS via anonymous ftp to cdsarc.u-strasbg. fr (130.79.128.5) or via http://cdsweb.u-strasbg.fr/cgi-bin/qcat?]/A+A/435/863 the sources can be classified based on their spectral shape. A spectral ageing analysis can reveal break and cut-off frequencies which eventually lead to the determination of particle ages, an important input to source evolution studies.

Nevertheless, there are particular spectral features like high-frequency spectral breaks, combinations of different spectral components or low-frequency turn-overs caused by synchrotron self-absorption (SSA) or free-free absorption which can often be detected only at the edges of the classical radio frequency range. Thus, we extended our database down to $74 \mathrm{MHz}$ using the observations made by Tschager et al. (2003, henceforth T03) which covered large areas of the B3 VLA region with a survey originally intended to determine spectral turn-overs in a sample of CSS sources. The common region contains 365 B3 VLA sources.

Throughout this paper we use $H_{0}=71 \mathrm{~km} \mathrm{~s}^{-1} \mathrm{Mpc}^{-1}$ and $q_{0}=0.5$, if not explicitly stated otherwise.

\section{The source extraction}

The observations were done with the VLA in its A-array at six pointings between $08^{\mathrm{h}}<\mathrm{RA}<17^{\mathrm{h}}$ and $37^{\circ}<$ Dec $<$ $43^{\circ}$ with a primary beam width of 11.7 . For the details of 
observations and data analysis we refer to T03. Starting from the calibrated radio maps we have used AIPS tasks IMFIT (for well-defined, almost point-like sources) and TVSTAT (for diffuse, complex sources) to obtain the integrated flux densities at the positions of the B3 VLA sources.

When using TVSTAT the local noise value $\left(\sigma_{1}\right)$ was determined in an annular area centred on the source but at a safe distance, thus carefully avoiding integration over confusing unrelated emission. The resulting contribution to the total measurement error was calculated as $\sigma_{1} \sqrt{A}$, where $A$ is the area (in units of beam solid angle) used in determining the flux density of the radio source. When using IMFIT the noise term was given by the fit error, which is on average three times greater than the local noise. In order to maximize the number of B3 VLA sources with a 74-MHz measurement we extended the extraction of flux densities out to a distance of 5.5 from the phase centre, where the primary beam is attenuated to $30 \%$. In this way we obtained 74-MHz flux densities (or upper limits) for all 365 B3 VLA sources in the observed area. Taking into account that $\mathrm{T} 03$ found a significant decrease of the source density at distances larger than $3^{\circ}$ from the phase centre of each pointing we have performed several tests to assure the quality of the extracted values.

\section{Data quality}

We have used two different approaches to test the quality of the calibrated data. First we compared our data with the 74-MHz data of the VLA Low-Frequency Sky Survey (VLSS), obtained with the VLA in B-array. In a second approach we used the known radio continuum data of the B3VLA survey to extrapolate the spectra to $74 \mathrm{MHz}$.

One of the problems of high-resolution observations at low frequencies is the calibration of the phase which often varies considerably due to the small size of the isoplanatic patch with respect to the field of view. This leads to apparent defocusing of sources - and decreasing peak flux densities - in a certain distance from the strong source used for phase calibration. Therefore, the angular extension of the B3 VLA radio sources measured with a simple Gaussian fit provides a rough quality check. Figure 1 shows the increase of the sources' major axes with distance from the phase centre for a representative field of T03. This will affect the flux-density measurements. In order to evaluate this effect we extracted the data from the VLSS (release of June 2002; Perley et al. 2003) in the area in common with the survey of T03. In the overlapping region, which is $2^{\circ}$ in declination, we found 38 radio sources. For these sources we computed the ratio of the flux densities of both surveys $\left(R=S_{(\mathrm{VLSS})} / S_{(\mathrm{T} 03)}\right)$. The median value of $R$ is $1.4 ; 31 \mathrm{ra}-$ dio sources have $R$ less than 2.5 while the remaining 7 have $R$ between 2.5 and 5. A sigma-clipping algorithm has been applied excluding 7 sources with $R$ larger than 2.5 , after which we obtained an average value $R=1.3 \pm 0.07$. No correlation between $R$ and the distance from the phase centre has been found, which is probably due to the relatively small range of distance from 3.5 to 6.3 investigated with this method.

In an alternative approach we have complemented the measured flux densities with the measurements of the B3VLA data

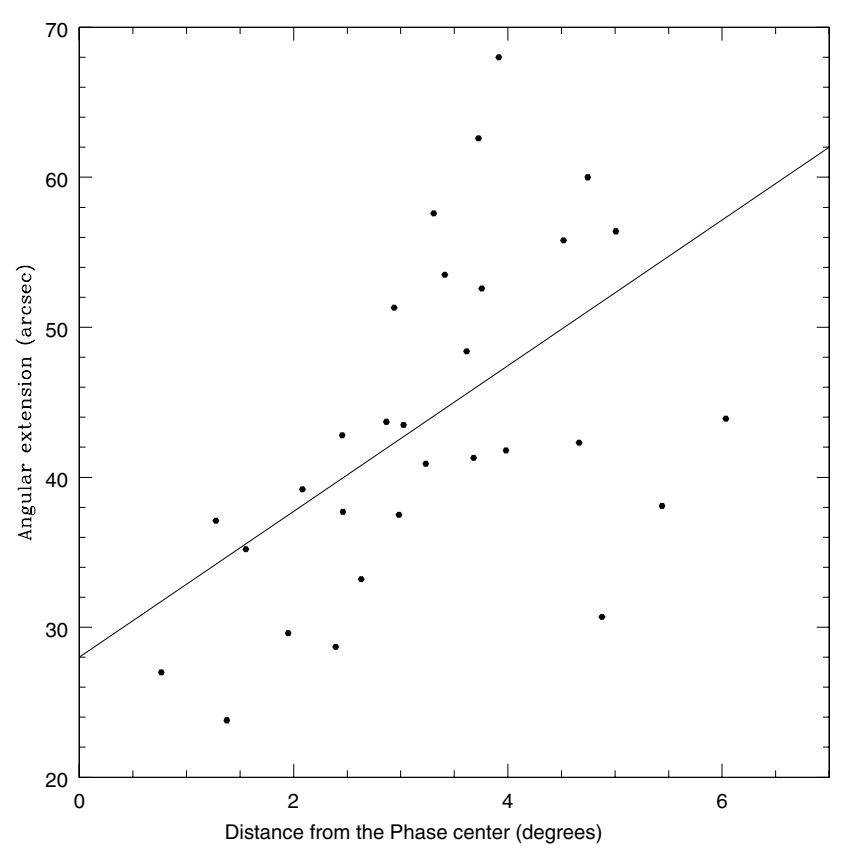

Fig. 1. Major axis (in arcsec) of the radio sources fitted with IMFIT versus distance (in degrees) from the phase centre for a representative field of $\mathrm{T} 03$.

base (Vigotti et al. 1999). Using standard synchrotron aging models like the continuous injection model (CI, Pacholzcyk 1970) and the Jaffe \& Perola (1974, JP) model we have extrapolated the flux densities to $74 \mathrm{MHz}$. Measurements marking spectral turnovers possibly caused by synchrotron selfabsorption or free-free absorption were flagged. 127 sources had significant $(S / N \geq 4) 74-\mathrm{MHz}$ detections and sufficiently reliable spectral fits $\left(\chi^{2} \leq 5\right)$ in order to be studied more thoroughly. Since any statistical deviation of the measured flux density $\left(S_{\text {mea }}\right)$ from the extrapolated one $\left(S_{\text {ext }}\right)$ in this subsample can be assumed to be caused by measurement errors we have quantified these discrepancies by $D=\frac{S_{\text {mea }}-S_{\text {ext }}}{S_{\text {ext }}}$. There is a clear trend of decreasing flux densities with increasing distance from the phase centre (Fig. 2). A fit to this trend shows that already at a distance of $3^{\circ}$ (the maximum distance used by T03) some $30 \%$ of the flux density has been lost. This confirms the correction factor of 1.3 derived from the comparison with the VLSS.

Therefore, we corrected the measured flux densities for these losses as a function of their distance from the phase centre. The flux densities together with their errors are compiled in Table 2. Sources for which the measured flux density turned out to be lower than its error are reported as upper limits. These were fixed as three times the local noise level. The total flux density errors have been computed as the quadratic sum of the noise term and the calibration term $(\Delta \mathrm{Cal}=18 \%)$. This latter was derived from the scattering around the corrected calibration factor (Fig. 2).

Similar to the work of Gregorini et al. (1998) we also determined the flux densities in the individual components of double sources which we compile in Table 3 for future reference. 


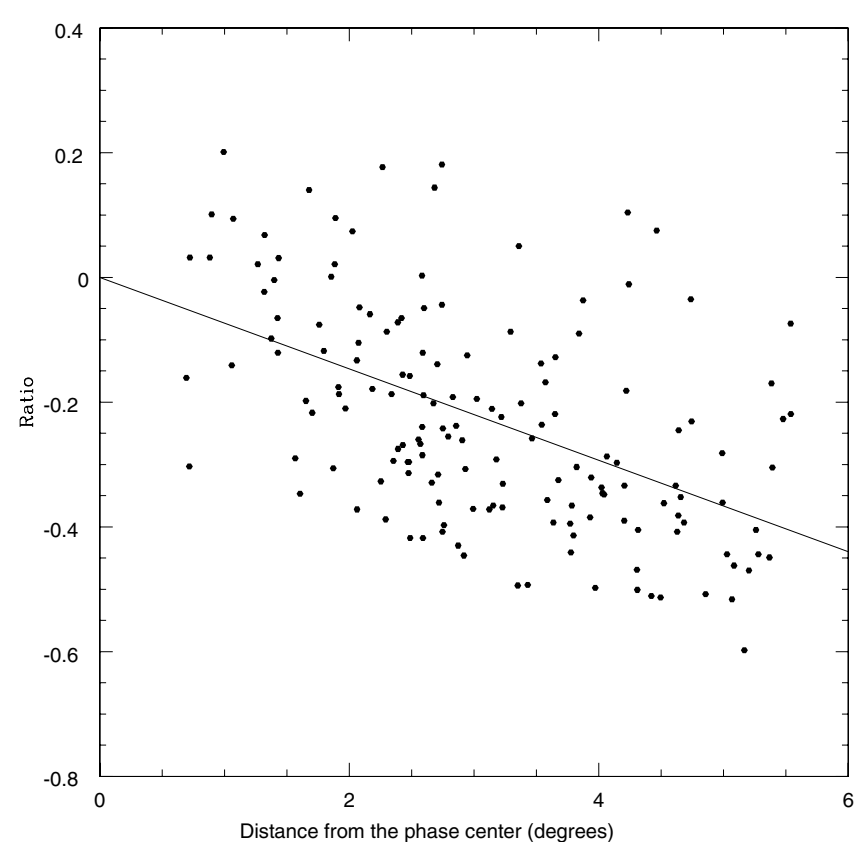

Fig. 2. Ratio $D$ (see text for definition) of measured and extrapolated flux densities (normalized by the extrapolated flux density) of 127 sources with significant $74-\mathrm{MHz}$ detections and well fitted synchrotron spectra. The straight line shows the fitted correction factor as a function of the sources' distance from the phase centre. This correction factor was subsequently applied to the data.

\section{Radio continuum spectra}

The corrected flux densities were further studied to reveal particular properties predominantly visible at the lowest radio frequencies. Excess flux densities could be an indication for a former activity period of the sources as suggested by Jamrozy et al. (2004) for the relic source B2 0924+24. On the other hand, flux densities which are lower than their model values could have been diminished by synchrotron self- or free-free-absorption. Of the 365 B3 VLA sources in the observed area, 294 sources were detected; for the rest we used the upper limits.

We have evaluated the fraction of sources with a lowfrequency turn-over using two different approaches. First, we fitted the optically thick parts of the spectra with a slope of $\alpha_{\text {thick }}=-2.5\left(S_{v} \propto v^{-\alpha}\right)$ as theoretically expected for synchrotron self-absorption and matched them to the optically thin part, either by a CI or a JP model. In a subsequent visual inspection we counted all those sources with a well-fitted turn-over frequency $>151 \mathrm{MHz}$, i.e. with at least two measurements confirming the low-frequency turn-over. This resulted in 19 out of 365 sources having a significant turn-over peak above $151 \mathrm{MHz}$ (i.e. $5 \%$ of the complete low-frequency selected sample).

Second, in a different approach we used the 160 brightest sources with $S_{408 \mathrm{MHz}} \geq 400 \mathrm{mJy}$, thus maintaining the completeness of the sample. We determined the deviations of the measured flux densities $S_{\text {mea }}$ at $74 \mathrm{MHz}$ from those extrapolated from the optically thin part of the spectrum $S_{\text {ext }}$. A histogram of these deviations $\Delta S=\frac{S_{\text {mea }}-S_{\text {ext }}}{S_{\text {ext }}}$ shows the

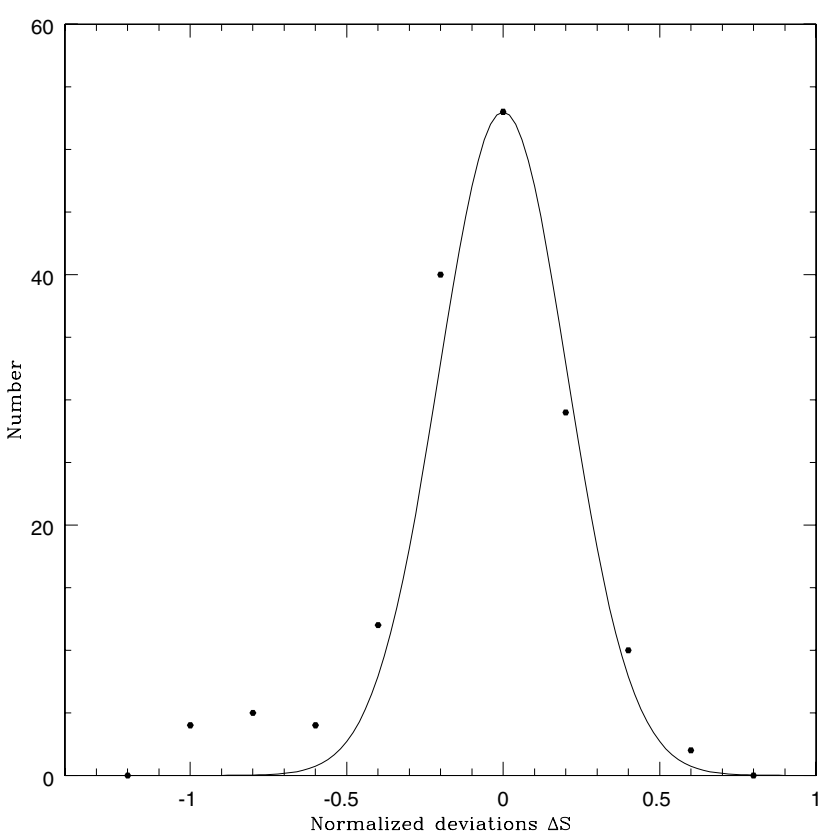

Fig. 3. Histogram of the deviations $\Delta S$ (see text for definition) of the measured 74-MHz flux densities from the predicted ones. In addition to the Gaussian distribution caused by measurement errors there is a number of sources with a flux density smaller than expected, which can be explained by absorption processes typically occurring in compact radio sources.

expected Gaussian distribution due to measurement errors, and in addition a wing of additional sources with $S_{\text {mea }}$ significantly smaller than $S_{\text {ext }}$ (Fig. 3). After determination of the Gaussian distribution by fitting a half-Gaussian to the $S_{\text {ext }}>S_{\text {mea }}$ part and applying the shape to the winged $\left(S_{\text {ext }}<S_{\text {mea }}\right)$ part of the distribution the number of sources with significantly smaller flux densities than expected can be determined. In total we find $25 \pm 5$, i.e. $(15.5 \pm 3.0) \%$, sources with clear indications of a turn-over at low frequencies.

These two different approaches give an indication of the lower and upper limits of turn-over sources in a low-frequency selected sample. Samples like the B3 VLA are supposed to contain the parent population of radio sources. The compactness of sources in such a sample can either be caused by young, thus genuinely compact like Giga-Hertz-Peaked (GPS) or Compact Steep Spectrum (CSS) sources or by beamed BL Lac or quasarlike sources which only appear to be compact. As we only looked at steep-spectrum sources to which the standard synchrotron ageing models can be applied, our results give an indication of the fraction of compact sources with significantly detected turn-over peaks. Because of the large uncertainty that we still have in the present data we refrain from drawing further conclusions at this point, although in principle this number is important for tests of the unified scheme, as it is a direct measure of the compactness in a sample thought to be free of orientational biases.

On the other hand, also by visual inspection, we have identified three sources the measured flux densities of which significantly $(>3 \sigma)$ exceed those extrapolated from 
the synchrotron ageing models. These are B3 1221+398, B3 1226+395 and B3 1411+397. The first two sources still lack a flux density measurement at $151 \mathrm{MHz}$; this excess can thus not be confirmed yet, B3 1411+397, however, shows a clear excess which is also confirmed by the 151-MHz measurement. This source is hosted by a galaxy at redshift of 0.328 and classified as resolved with an angular size of $19^{\prime \prime}$ by Vigotti et al. (1989). Deep high-resolution observations are required to investigate the mixture of relic and recurrent activity likely to be present in this source.

\section{Morphologies}

We compared the angular sizes of the 294 B3 VLA sources detected at $74 \mathrm{MHz}$ with those at higher frequencies. Because the actual beamsize at 74-MHz changes from field to field as well as with distance from the field centre we have always calibrated the source extents with the "local" beam sizes, i.e. with the angular size of a point-like source within a radius of $1^{\circ}$ around the target source. In order to study the abundance of sources with unusually steep spectra, we have extended our search to all sources with extents larger than 24" (corresponding to the nominal beam width).

Therefore, we deconvolved the target sources with the local beam size, derived from a fit to nearby strong point-like sources in order to find the significantly extended sources; these are listed in Table 1 with their B3VLA names. In the following we shall comment on the four most remarkable of these sources. For these four sources we have used the 1.4-GHz maps of the NVSS to calculate the spectral index distribution between $74 \mathrm{MHz}$ and $1.4 \mathrm{GHz}$. In doing this we have smoothed the $74-\mathrm{MHz}$ map to the NVSS resolution of $45^{\prime \prime}$. Surveys at lower frequencies than the NVSS, e.g. WENSS, have larger beamsizes $\left(55^{\prime \prime} \times 85^{\prime \prime}\right.$ in the case of the WENSS) and hence do not provide sufficiently resolved images for a comparison with the 74-MHz A-array data.

\section{B3 0938+399B (3C 223.1):}

This radio galaxy at redshift 0.1075 belongs to the class of $\mathrm{X}$-shaped radio galaxies and has been thoroughly studied by various authors (e.g. Rottmann 2001; Dennett-Thorpe et al. 2002, and references therein). X-shaped or winged radio galaxies are formed by two double-lobe systems emanating at clearly distinct position angles from the source centres. It is assumed that this morphological peculiarity is an indication of different activity periods of their central machines. Unfortunately, in the spectral index map with its resolution of $45^{\prime \prime}$ there are hardly any independent pixels left which cover the wings of B30938+399B (Fig. 4). However, the tendency of the spectral index distribution is in line with the findings of Dennett-Thorpe et al. (2002), reported in their Table 4. Also in our case the few significant spectral indices in the - presumably older - wings seem to be slightly flatter than in the active lobes. Due to the large span in frequency between $74 \mathrm{MHz}$ and $1.4 \mathrm{GHz}$ calibration errors of $\sim 20 \%$ would yield errors in our spectral indices of only \pm 0.06 . Loss of flux density due to missing short-spacings should affect both data sets (A-array at $74 \mathrm{MHz}$ and D-array at $1.4 \mathrm{GHz}$ )
Table 1. Extended B3 VLA sources at $74 \mathrm{MHz}$.

\begin{tabular}{llll}
\hline \hline $0938+399 \mathrm{~B}$ & $0954+436$ & $1004+446$ & $1007+417$ \\
$1009+434$ & $1013+410$ & $1014+397 \mathrm{~A}$ & $1019+394$ \\
$1020+400$ & $1025+390 \mathrm{~B}$ & $1039+424$ & $1041+392$ \\
$1219+382$ & $1232+397 \mathrm{~B}$ & $1359+419$ & $1411+397$ \\
$1419+419$ & $1424+380$ & $1447+380$ & \\
\hline
\end{tabular}

in a similar way but can probably be neglected anyway. The straight power-law spectra seem to continue down to $74 \mathrm{MHz}$ without any curvature which confirms the break frequencies fitted by Dennett-Thorpe et al. to lie at frequencies $\geq 12 \mathrm{GHz}$.

\section{B3 1013+410 (4C 41.22):}

This source, hosted by a galaxy at redshift $z=0.117$ and classified as a double with a total angular size of $122^{\prime \prime}$ in the B3 VLA (Vigotti et al. 1989), shows three components at $74 \mathrm{MHz}$ (Fig. 5). The spectral index distribution between $74 \mathrm{MHz}$ and $1.4 \mathrm{GHz}$ reveals the third, central component as a steep-spectrum $(\alpha \sim 1.1)$ core. Steep-spectrum cores, while relatively common in high-redshift radio galaxies where the optically thin part of the synchrotron spectrum is redshifted towards cm-wavelengths (Athreya et al. 1997), are much more rarely found at low redshifts and at longer wavelengths. The most probable explanation is the presence of an underlying structure, not resolved by a beamsize of $25^{\prime \prime}$. In fact, the FIRST map shows a slightly extended central core which could host several steep-spectrum components or be caused by a "halo" of extended emission around a weaker flat-spectrum compact core (Saikia et al. 1986). Higher-resolution observations are required to disclose the origin of this steep-spectrum core.

\section{B3 1232+397B (4C 39.37):}

This galaxy has the highest known redshift of the B3 VLA sample, $z=3.22$ (Eales et al. 1993). It is characterized by a very steep spectrum, even at very low frequencies $(\alpha \simeq 1)$. Our 74-MHz map shows two protrusions in the NW-SE direction which are not seen at higher frequencies (Fig. 6). As it cannot be excluded that these are artefacts caused by an imperfect amplitude calibration, deep low-frequency observations are required to verify these features. Our $74-\mathrm{MHz}-1.4-\mathrm{GHz}$ spectral index map can only give an indication of the spectral indices in the winged structure. The last significant pixels imply $\alpha>1$.6. Probably spectral indices of $\alpha \sim 1.9$ are reached further out, considering that the $1.4-\mathrm{GHz}$ emission is below the NVSS noise limit of $0.45 \mathrm{mJy} / \mathrm{beam}$. Carilli et al. (1997) obtained high-resolution maps of the central component which turns out to be formed by a radio galaxy of $\sim 8^{\prime \prime}$ angular size, while at $74 \mathrm{MHz}$ we find an angular extent of about 2'2. At the source's redshift of 3.22 this translates to a linear size of $1 \mathrm{Mpc}\left(930 \mathrm{kpc}\right.$ with $H_{0}=50 \mathrm{~km} \mathrm{~s}^{-1} \mathrm{Mpc}^{-1}$ ). B3 1232+397B is thus the most distant Giant Radio Galaxy (GRG) found so far. Carilli et al. report double hot spot systems which they interpret as an indication of a change of the jet axis during the source's lifetime. This finding, together with the existence of the steep-spectrum wings, let us speculate that 

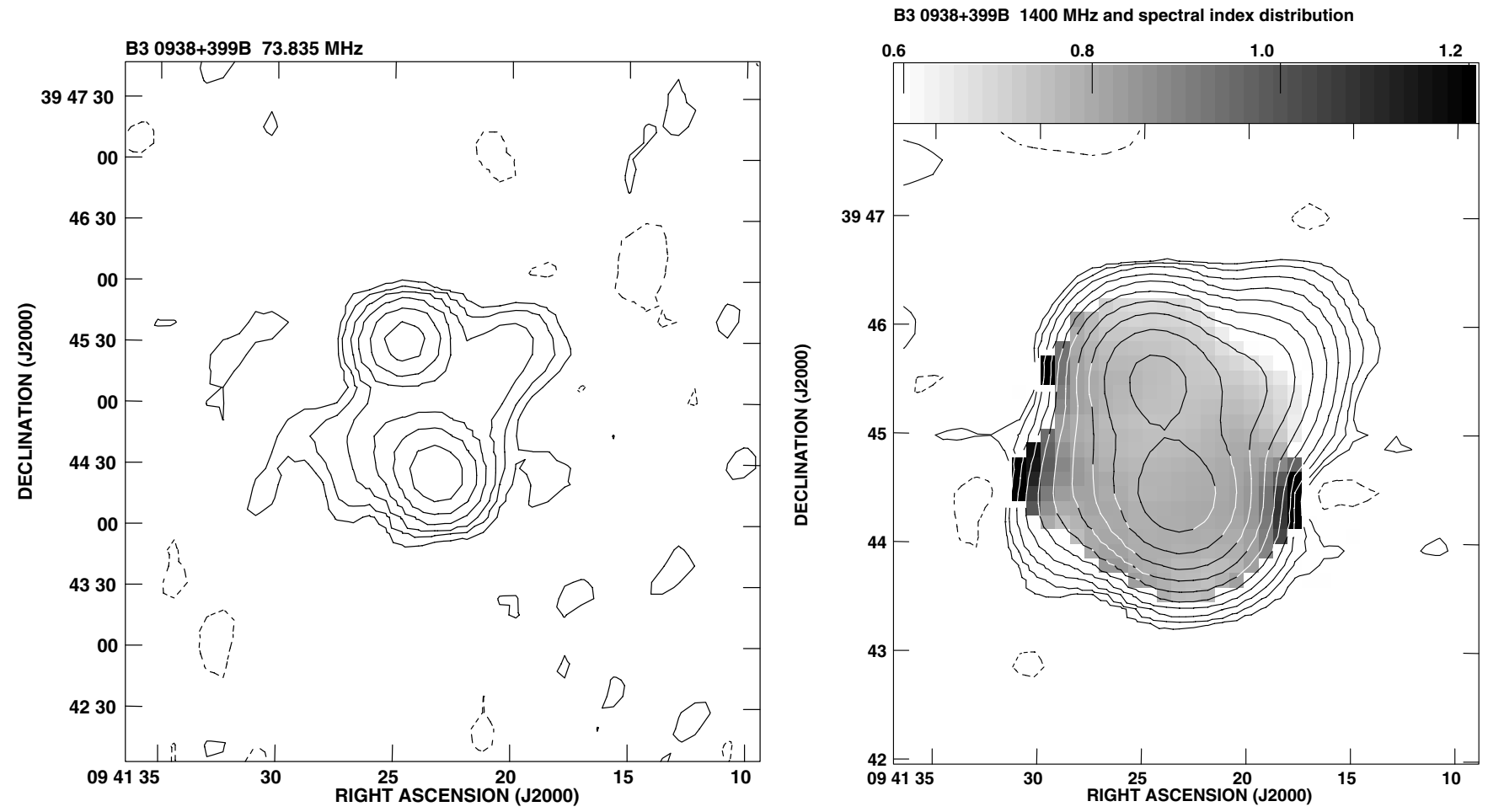

Fig. 4. B3 0938+399B: left: 74-MHz map, contour levels: $-0.1,0.1$, ,0.2, 0.4, 0.8, 1.6, $3.2 \mathrm{Jy} /$ beam; right: spectral index distribution (grey), NVSS flux densities (contours), contour levels: $-1,1,2,4,8,16,32,64,128,256,512,1024,2048 \mathrm{mJy} / \mathrm{beam}$.
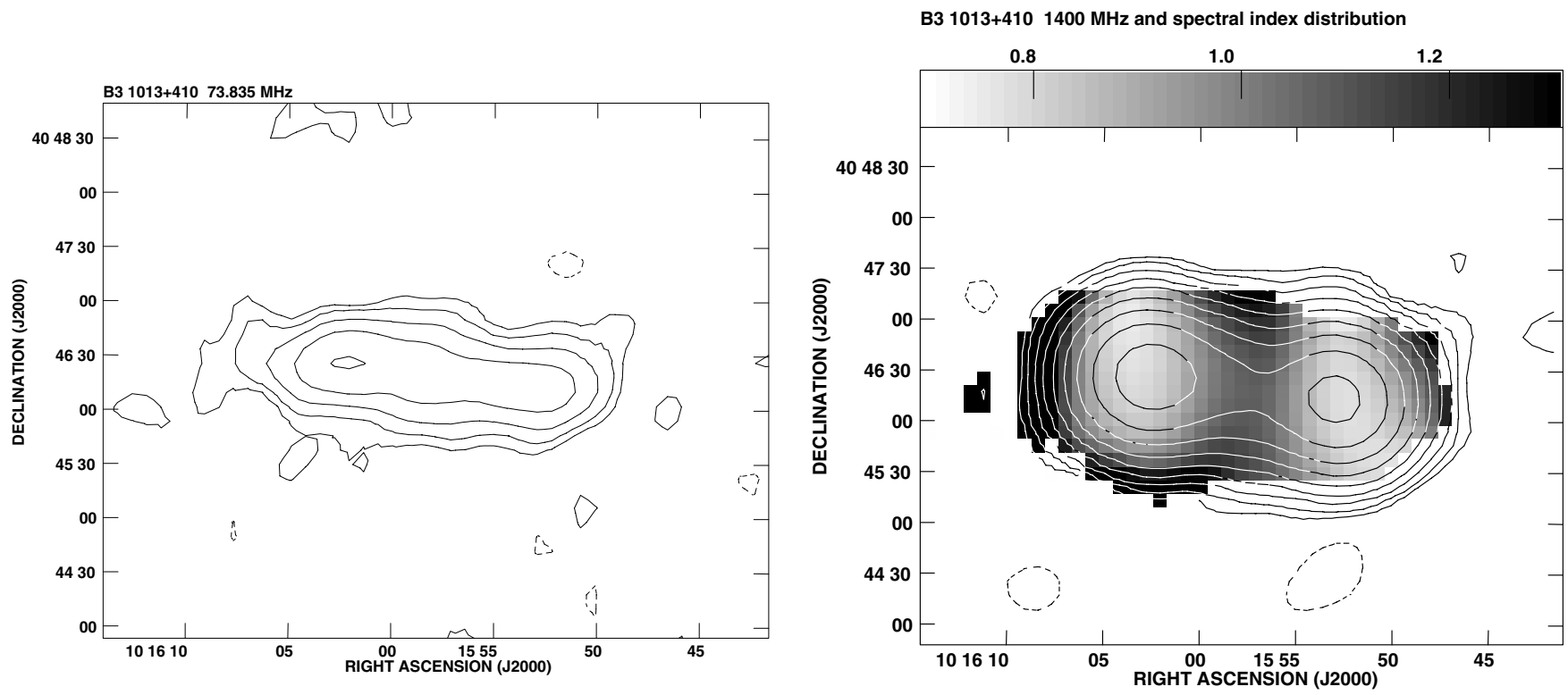

Fig. 5. B3 1013+410: left: 74-MHz map, contour levels: -0.1, 0.1, 0.2, 0.4, 0.8, 1.6 Jy/beam; right: spectral index distribution (grey), NVSS flux densities (contours), contour levels: $-1,1,2,4,8,16,32,64,128,256$ mJy/beam.

this source underwent several periods of recurrent activity in the past. The extended structure that we found at $74 \mathrm{MHz}$ would then reflect the relic of a previous activity phase. This would classify B3 1232+397B also as a Double-Double Radio Galaxy (Schoenmakers et al. 2000).

B3 1419+419 (3C 299):

This source has been classified as a Compact Steep Spectrum source by various authors. Similar to B3 1232+397B, the 74-MHz map reveals a diffuse structure extended on both sides of the core reminiscent of a relic lobe system which has not been seen at other frequencies. WENSS data show only a faint indication, if any, of these wings. As the noise level of the WENSS in this area is $\sim 3 \mathrm{mJy} /$ beam, this means an extremely steep spectral index of $\alpha_{74}^{325} \sim 3$. In fact, our spectralindex map (Fig. 7) shows $\alpha \sim 2$ in the outermost pixels which are still significant in the $1.4-\mathrm{GHz}$ map, while the centre has the typical spectral index of a standard active radio source 

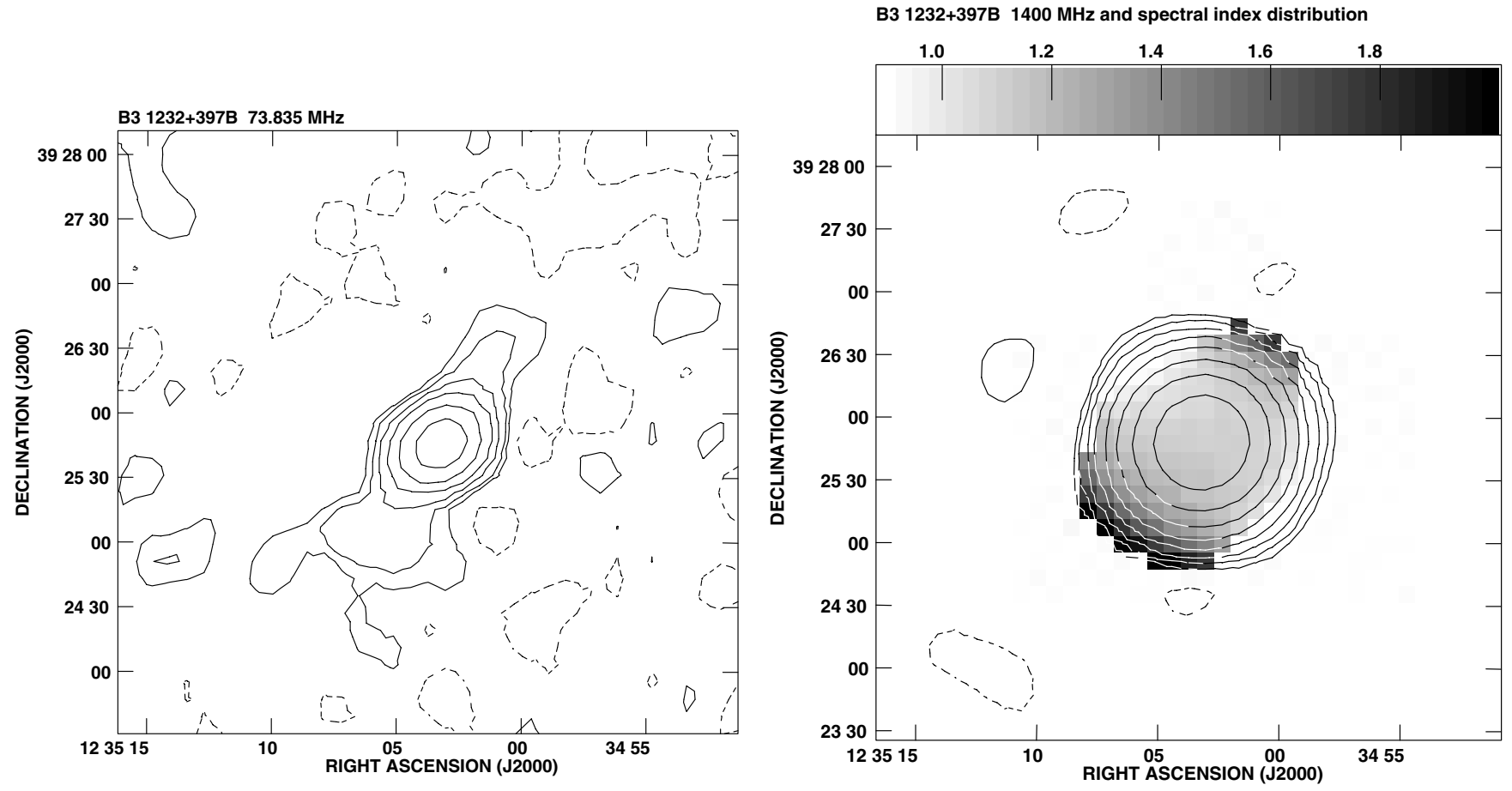

Fig. 6. B3 1232+397B: left: 74-MHz map, contour levels: -0.1, 0.1, 0.2, 0.4, 0.8, 1.6, $3.2 \mathrm{Jy} / \mathrm{beam}$; right: spectral index distribution (grey), NVSS flux densities (contours), contour levels: $-1,1,2,4,8,16,32,64,128 \mathrm{mJy} / \mathrm{beam}$.
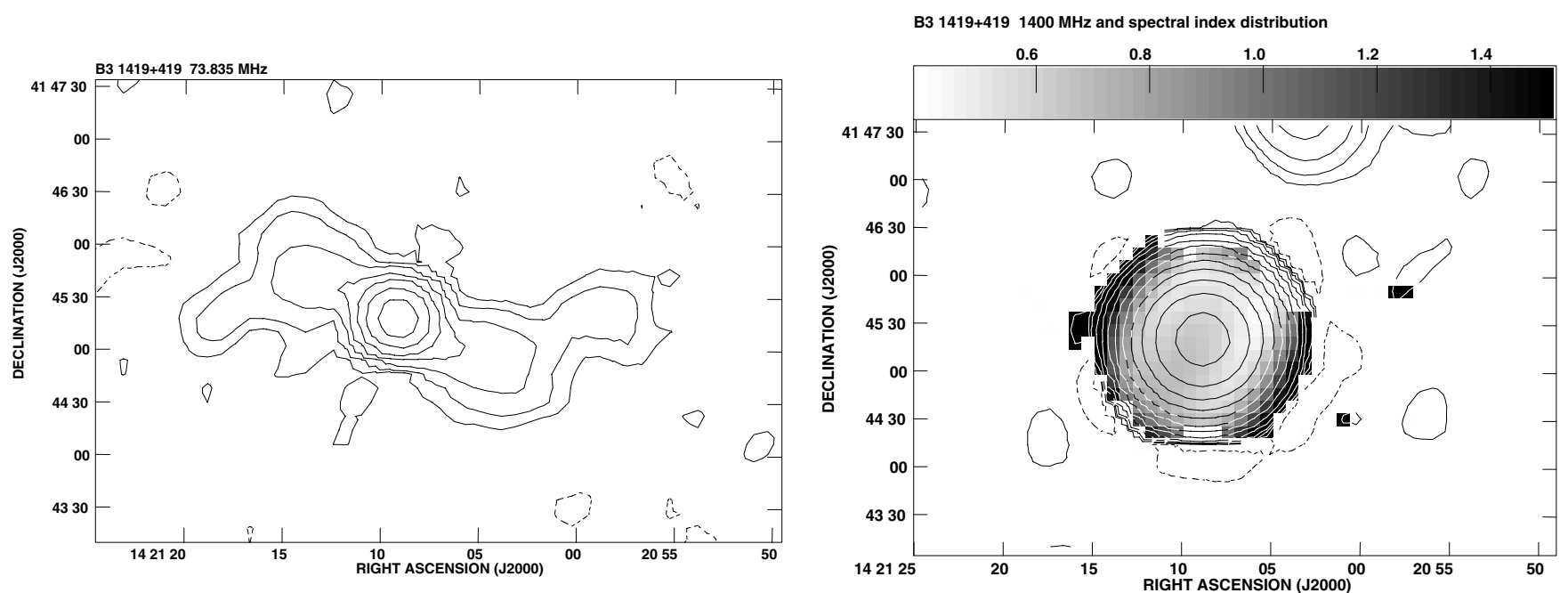

Fig. 7. B3 1419+419: left: 74-MHz map, contour levels: -0.2, 0.2, 0.4, 0.8, 1.6, 3.2, 6.4, $12.8 \mathrm{Jy} /$ beam; right: spectral index distribution (grey), NVSS flux densities (contours), contour levels: -1, 1, 2, 4, 8, 16, 32, 64, 128, 256, 512, 1024, 2048 mJy/beam.

$(\alpha \approx 0.5-0.7)$. This morphology not only ranks B3 1419+419 in the class of sources with recurrent activity, but its extended features must be regarded as being the oldest fossil structures detected around young radio galaxies. The total extent of the radio structure of at least 3.2 corresponds to $970 \mathrm{kpc}(1.2 \mathrm{Mpc}$ with $\left.H_{0}=50 \mathrm{~km} \mathrm{~s}^{-1} \mathrm{Mpc}^{-1}\right)$. This means that B3 $1419+419$ is also a GRG and - compared to the presently known GRGs at the relatively high redshift of 0.367 . This recurrent activity scenario - a young source in the centre surrounded by remains from a previous activity period - is also suggested by optical HST images (de Koff et al. 1996). The host galaxy is distorted, showing three patches of emission and copious filamentary emission, which could be indications of a recent merger which caused the onset of the present radio-active phase.

\section{Prospects for LOFAR}

In this work, we have identified a number of sources exhibiting extended emission, which in some cases is not seen at higher frequencies. It is here that the potential power of low-frequency radio astronomy is revealed: future observations with LOFAR will bring a rich harvest of diffuse, extended radio continuum structures not visible at $\mathrm{cm}$ wavelengths, owing to their steep 
spectra. This implies that we are dealing with aged synchrotron emitters, such as quenched radio galaxies or relics ${ }^{1}$.

There are three kinds of aged radio sources that will be found. The first is simply comprised by aged components of radio galaxies that can still be identified with a host galaxy. Such sources are either starved AGN (e.g. Jamrozy et al. 2004), or they contain distinct radio components attributable to different epochs of activity. Such repetitive activity has already been noted in a number of radio galaxies, sometimes producing self-similar structures, the so-called "double-double" radio galaxies (see Schoenmakers et al. 2000), sometimes exhibiting a change in their orientation, thus producing a species referred to as "X-shaped" radio galaxies, or more complex sources such as Vir A (see e.g. Klein 1999). It is to be expected that LOFAR will disclose numerous steep-spectrum, extended structures of the above kind, associated either with known radio sources or (predominantly) with elliptical galaxies that do not exhibit any signs of current activity.

The second kind of steep-spectrum, extended radio emission to be disclosed at low frequencies are the so-called cluster halos. These are large, diffuse radio continuum structures found in the centres of galaxy clusters, their exact origin still being a matter of speculation. Their most likely origin is turbulent reacceleration of a cooled pool of relativistic electrons (Brunetti 2003). About two dozen such cases are known to date (Feretti 2003; Sarazin 2002).

The third kind are peripheral radio structures in clusters of galaxies, most likely generated by the merging of two galaxy clusters, whereby magnetic fields are compressed and amplified and particles (re-)accelerated to highly relativistic energies. It should be noted that intra-cluster turbulence may also be responsible for the long extensions of tailed radio sources seen at low frequencies (3C 465: Sijbring \& de Bruyn 1998; 3C 129: Lane et al. 2002). These low-frequency tails can neither be explained in terms of ballistic orbits of their host galaxies in the cluster potentials, neither in terms of synchrotron lifetimes.

Finally, we point out that the detection of diffuse lowfrequency radio continuum structures requires excellent imaging. Since ionospheric phase errors scale with wavelength squared, this clearly requires new calibration and imaging strategies. Such procedures are currently being developed and applied to the VLSS and include the correction of ionospheric refraction errors and the compensation of non-coplanarity of the VLA (Perley et al. 2003). It is obvious that with the relatively poor imaging quality achieved in the current work, many such structures would escape detection.

\section{Summary}

We have used the data presented by $\mathrm{T} 03$, obtained with the VLA in the A-array to obtain flux densities and morphological information for 365 B3 VLA sources at $74 \mathrm{MHz}$. In total, 294 sources were detected at least at a $3 \sigma$-level, while for the remaining 71 sources upper limits could be established. Several

\footnotetext{
1 The reader is referred to the clarifying paper by Kempner et al. (2003) regarding the taxonomy and likely physical conditions of the various diffuse radio sources in galaxy clusters.
}

tests were applied to investigate the quality of the derived flux densities and to improve their calibration. This could be done because the radio continuum spectra of the B3 VLA sources have been well measured between $151 \mathrm{MHz}$ and $10 \mathrm{GHz}$, thus allowing a reliable extrapolation down to $74 \mathrm{MHz}$ in many cases. We also used the information available in the VLSS data (release of June 2002), which has a small overlap with the data used in this work.

Since no further selection criterion has been applied, we can consider the sample as statistically complete. Using two different approaches we have determined the fraction of sources with low-frequency turn-overs in this low-frequency selected sample to lie between 5\% and $15 \%$. This turn-over is caused by absorption processes (synchrotron self- or thermal free-free absorption) in compact sources.

Furthermore we have looked at extended sources. Among the 19 sources which were found to be significantly more extended than the beamsize, we found two new giant radio galaxy candidates, one of which, B3 1232+397B, at $z=3.22$, would be the highest redshift found for GRGs so far. Remarkably, all existing low-frequency surveys missed the extended emission around these sources of which up to date only the bright centres were known. The reason for this lies in the extremely steep spectral indices of the extended regions. Both sources have been classified as CSS, thus presumably being young sources. Now, the newly found extended emission indicates a long-lasting previous active period which gave rise to the creation of a GRG.

These findings give a foretaste of what can be expected from very low-frequency observations as will be feasible with LOFAR.

Acknowledgements. We thank Dr. Maria Marchã for helpful discussions. The National Radio Astronomy Observatory is operated by Associated Universities, Inc., under cooperative agreement with the National Science Foundation.

\section{References}

Athreya, R. M., Kapahi, V. K., McCarthy, P. J., \& van Breugel, W. 1997, MNRAS, 289, 525

Brunetti, G. 2003, in Matter and Energy in Clusters of Galaxies, ed. S. Bowyer, \& C.-Y. Hwang, ASP Conf. Proc., 301, 349

Carilli, C. L., Röttgering, H. J. A., van Ojik, R., Miley, G. K., \& van Breugel, W. J. M. 1997, ApJS, 109, 1

de Koff, S., Baum, S. A., Sparks, W. B., et al. 1996, ApJS, 107, 621

Dennett-Thorpe, J., Scheuer, P. A. G., Laing, R. A., et al. 2002, MNRAS, 330, 609

Eales, S. A., Rawlings, S., Dickinson, M., et al. 1993, ApJ, 409, 578

Feretti, L. 2003, in Matter and Energy in Clusters of Galaxies, ed. S. Bowyer, \& C.-Y. Hwang, ASP Conf. Ser., 301, 143

Ficarra, A., Grueff, G., \& Tomassetti, G. 1985, A\&AS, 59, 255

Gregorini, L., Vigotti, M., Mack, K.-H., Zönnchen, J., \& Klein, U. 1998, A\&AS, 133, 129

Jaffe, W. J., \& Perola, G. C. 1973, A\&A, 26, 423

Jamrozy, M., Klein, U., Mack, K.-H., Gregorini, L., \& Parma, P. 2004, A\&A, 427, 79

Kempner, J. C., Blanton, E. L., Clarke, T. E., et al. 2003, Proc. The Riddle of Cooling Flows in Galaxies and Clusters of Galaxies, ed. T. Reiprich, J. Kempner, N. Soker

(http://www. astro.virginia.edu/coolflow/) 
Klein, U. 1999, in The Radio Galaxy Messier, ed. H.-J. Röser, \& K. Saikia, D. J., Kulkarni, V. K., \& Porcas, R. W. 1986, MNRAS, 219, Meisenheimer (New York: Springer), 87, 56

Klein, U., Vigotti, M., Gregorini, L., et al. 1996, A\&A, 313, 417

Klein, U., Mack, K.-H., Gregorini, L., \& Vigotti, M. 2003, A\&A, 406, 579 719

Sarazin, C. L. 2002, in Merging Processes in Clusters of Galaxies, ed. L. Feretti, I. M. Gioia, \& G. Giovannini, Astrophys. Space Sci. Lib., 272, 1

Lane, W. M., Kassim, N. E., Enßlin, T. A., Harris, D. E., \& Perley, R. A. 2002, AJ, 123, 2985

Pacholczyk, A. G. 1970, Radio Astrophysics (San Francisco: Freeman)

Perley, R. A., Condon, J. J., Cotton, W. D., et al. 2003, URL: http://lwa.nrl.navy.mil/VLSS/

Rottmann, H. 2001, Ph.D. Thesis, Univ. Bonn (http://hss.ulb.uni-bonn.de/diss_online/ math_nat_fak/2001/rottmann_helge/index.htm)

Schoenmakers, A. P., de Bruyn, A. G., Röttgering, H. J. A., van der Laan, H., \& Kaiser, C. R. 2000, MNRAS, 315, 371

Sijbring, D., \& de Bruyn, A. G. 1998, A\&A, 331, 901

Tschager, W., Schilizzi, R. T., Röttgering, H. J. A., et al. 2003, A\&A, 402, 171 (T03)

Vigotti, M., Gregorini, L., Klein, U., \& Mack, K.-H. 1999, A\&AS, 139,35

Vigotti, M., Grueff, G., Perley, R., Clark, B. G., \& Bridle, A. H. 1989, AJ, 98, 419 